\title{
Teońa Económica y Credibilidad en la Política Monetaria
}

\author{
Moisés J. Schwartz y Sybel Galván ${ }^{1}$
}

\author{
Marzo de 1999 \\ D ocumento de Investigación No. 9901 \\ Dirección General de Investigación Económica \\ BANCO DE MÉXICO
}

1Las opiniones contenidas en este trabajo corresponden exclusivamente a los autores y no necesariamente representan el punto de vista del Banco de México. 


\title{
Teonía Económica y Credibilidad en la Política Monetaria
}

\author{
Moisés J. Schwartz y Sybel G alván \\ Marzo de 1999 \\ D ocumento de Investigación No. 9901
}

\begin{abstract}
RESUMEN
La instrumentación de la política monetaria, como la de cualquier otra política económica, puede describirse como un proceso continuo de interacción entre las autoridades y el público. En dicho proceso secuencial, las autoridades determinan y revisan sus políticas, así como sus instrumentos, a la vez que el público se adapta y anticipa al comportamiento de la autoridad. D e esta manera, los resultados de política pueden describirse como equilibrios endógenos que resultan del proceso de interacción entre los hacedores de política y los demás agentes económicos. El propósito de este trabajo es presentar algunas consideraciones teóricas sobre la forma en que los bancos centrales pueden mejorar su credibilidad, y por consiguiente facilitar el proceso de reducción de la inflación.
\end{abstract}




\section{Teońa Económica y Credibilidad en la Política Monetaria}

\section{Introducción}

D urante los últimos años la independencia de la banca central se ha convertido en una especie de norma para la organización y el funcionamiento de los bancos centrales en un número importante de países. Así, a través de modificaciones legales y resoluciones jurídicas, e inclusive constitucionales, diversos países le han otorgado a sus bancos centrales la independencia legal con respecto a otros sectores de la administración pública, con el objeto de que las autoridades monetarias velen, primordialmente, por la estabilidad de la moneda. $\mathrm{Si}$ bien algunos países no han realizado las adecuaciones jurídicas que determinen legalmente la independencia de sus bancos centrales, éstos han mostrado un importante cambio de actitud con relación a los objetivos y a las facultades de sus respectivas autoridades monetarias. En particular, en tiempos recientes, se ha observado una clara orientación de la política monetaria hacia el objetivo de estabilidad de precios en prácticamente todos los países. Por otra parte, a la par en que el mundo ha evidenciado la evolución de la banca central hacia esquemas de mayor independencia, la teoría económica también ha avanzado en la dirección de proponer la independencia de la autoridad monetaria como mecanismo formal para aumentar la credibilidad del compromiso antiinflacionario de la autoridad monetaria. La evidencia empírica, así como la teoría económica, presentan de manera elocuente que mientras mayor sea la credibilidad del público en el compromiso de abatimiento de la inflación de la autoridad, más fácilmente podrá alcanzarse el objetivo de estabilidad de precios.

La instrumentación de la política monetaria, como la de cualquier otra política económica, puede describirse como un proceso continuo de interacción entre las autoridades y el público. En dicho proceso secuencial, las autoridades determinan y revisan sus políticas, así como sus instrumentos, a la vez que el público se adapta y anticipa al comportamiento de la autoridad. De esta manera, los resultados de política pueden describirse como equilibrios endógenos que resultan del proceso de interacción entre los hacedores de política y los demás agentes económicos. El propósito de este trabajo es presentar algunas consideraciones teóricas sobre la forma en que los bancos centrales pueden mejorar su credibilidad, y por consiguiente facilitar el proceso de reducción de la inflación. Para tal propósito, la siguiente sección presenta algunos conceptos básicos sobre la independencia de la banca central y el proceso inflacionario. La tercera sección describe en forma detallada el desarrollo del sustento teórico a favor de la independencia de la autoridad monetaria como mecanismo idóneo para aumentar 
la credibilidad de los bancos centrales. La última sección presenta algunas consideraciones sobre la forma en que las autoridades monetarias pueden aumentar su credibilidad.

\section{Independencia de la Banca Central e Inflación}

Tanto académicos como autoridades han llegado a la conclusión de que aislar a la política monetaria de las decisiones políticas facilita la consecución de la estabilidad de precios, y permite que ésta se alcance a un menor costo en términos de actividad económica y empleo. Dicha conclusión se basa en el convencimiento de que la mejor manera en que la política monetaria puede servir al interés público, es propiciando un ambiente monetario y financiero estable para el desarrollo de la actividad económica, mediante la instrumentación de una política monetaria orientada al abatimiento de la inflación. Con base en lo anterior, un número importante de países le ha otorgado independencia a sus bancos centrales, asignándoles el mandato de encaminar su política hacia la consecución de la estabilidad de precios $^{2}$.

Es bien sabido que la inflación genera un sinnúmero de costos para el adecuado funcionamiento de una economía. La incertidumbre y la volatilidad que caracterizan al proceso inflacionario se reflejan en la ineficiente asignación de recursos productivos, tanto entre sus distintos usos como en el tiempo, y en una inequitativa distribución del ingreso ${ }^{3}$. No obstante, aun en el caso de que la inflación fuera perfectamente anticipada por el público, ésta genera costos en bienestar tales como los asociados al ajuste continuo de los precios de los bienes y servicios, al aumento de los costos de transacción en operaciones financieras y comerciables, y al incremento del costo de mantener dinero.

La conveniencia de contar con bancos centrales autónomos que tengan como su objetivo prioritario la procuración de la estabilidad de precios, se sustenta en dos hechos estilizados: a) la curva de Phillips de largo plazo es vertical, lo cual implica que en ese plazo la inflación no tiene un efecto permanente sobre el empleo, y b) los gobiernos tienen incentivos a generar

\footnotetext{
${ }^{2} \mathrm{~A}$ lo largo del documento el concepto de independencia de la banca central se refiere a la "independencia de instrumentos", lo cual implica que la autoridad monetaria elegirá por si misma, aquellos instrumentos que considere como los más idóneos para alcanzar sus objetivos. El concepto de "independencia de objetivos", que se refiere a que la propia autoridad monetaria establece sus metas, no es analizado en este trabajo.

${ }^{3}$ Ver por ejemplo, Fischer (1993), Bruno y Easterly (1995) y Martínez (1998).
} 
sorpresas inflacionarias, debido a la posible existencia de una curva de Phillips de corto plazo la cual implica, que para dicho plazo, pudiese existir la posibilidad de reducir el nivel de desempleo a cambio de una mayor inflación. Por otra parte, el contar con bancos centrales independientes también se sustenta en el hecho, de que a través de dicha modificación institucional, la autoridad monetaria adquiere una fórmula mediante la cual logra comprometerse en forma creíble a instrumentar una política cuyo objetivo sea mantener el poder adquisitivo de la moneda ${ }^{4}$.

Una de las relaciones de comportamiento más entendidas en la teoría monetaria es la que relaciona, en el mediano y largo plazo, a la tasa de inflación con la tasa de crecimiento económico y con la tasa de expansión de la oferta monetaria. Esta relación asevera que la tasa de crecimiento de la oferta monetaria tiene como contrapartida alguna tasa de crecimiento económico y cierto nivel de inflación. Vista desde otra perspectiva, la relación señalada indica que el crecimiento porcentual del nivel de precios resulta igual a la tasa de expansión monetaria que excede las necesidades de financiamiento que requiere el crecimiento potencial de la economía ${ }^{5}$. Esta relación de comportamiento se presenta en el mediano y largo plazo en cualquier economía, sin importar su estructura, ni su apertura comercial, ni su régimen cambiario, ni tampoco su estrategia monetaria ni las características propias del mecanismo de transmisión de la política monetaria ${ }^{6}$. Así, esta relación ha dado lugar a establecer que la inflación es siempre y en todo lugar un fenómeno monetario ${ }^{7}$.

No obstante lo anterior, existen en el corto plazo, factores ajenos al control directo de los bancos centrales que afectan la evolución de los precios y que dificultan la consecución de la estabilidad de precios. Entre estos factores destacan el comportamiento de los agentes económicos en la determinación de los precios y los salarios, los movimientos del tipo de cambio, las perturbaciones sobre la oferta y la demanda agregada y el manejo de las finanzas públicas, entre otros. Inclusive, factores subjetivos tales como las propias expectativas inflacionarias del público tienen un impacto sobre la evolución de los precios.

Más aún, en el corto plazo, los factores señalados pueden llegar a tener una mayor influencia sobre la inflación que la propia evolución de los agregados monetarios, ya que la política

\footnotetext{
${ }^{4}$ En términos más técnicos, dicha fórmula puede describirse como una "tecnología de compromiso".

${ }^{5}$ A sumiendo una velocidad de circulación del dinero constante.

${ }^{6}$ Las circunstancias actuales, las cuales se han caracterizado por la desregulación y la innovación financiera, han dado lugar a que algunos agregados monetarios satisfagan la relación señalada mejor que otros. No obstante, no queda duda alguna de que el adecuado manejo monetario es un requisito indispensable para abatir la inflación.
} 
monetaria actúa con rezago sobre el comportamiento de los precios. D ebido a la complejidad que caracteriza al proceso inflacionario, y a los distintos elementos que lo determinan, una adecuada instrumentación de la política monetaria resulta esencial, más no suficiente, para conseguir avances importantes en la lucha antiinflacionaria.

La independencia de la banca central es un elemento que propicia un prudente manejo monetario, y por consiguiente favorece el abatimiento de la inflación. Sin embargo, el éxito sostenible en la lucha contra la inflación requiere, además de un adecuado manejo monetario, de la concurrencia de los distintos sectores de la sociedad. Así, se requiere, entre otros factores, de la instrumentación de políticas fiscales sólidas, del convencimiento de las autoridades que abatir la inflación es deseable y que sus acciones reflejen la importancia de alcanzar dicho objetivo, de la cooperación de otras medidas de política, y del convencimiento de la población en general de que abatir la inflación redundará en beneficios para toda la sociedad. Por otra parte, las distorsiones y rigideces de algunos mercados pueden obstruir el proceso antiinflacionario, por lo que las reformas estructurales que propician la eficiencia en los distintos mercados, facilitarán el logro de la estabilización de precios.

Existe también consenso de que mientras más rápidamente ajusten los agentes económicos sus expectativas de inflación, y mientras más flexibilidad exista en los distintos precios de una economía, más fácil será para el banco central disminuir la inflación sin generar trastornos en la actividad económica. Es decir, menor será el "cociente de sacrificio" ${ }^{8}$. Esto último debido a que mientras más convencido se encuentre el público de que la autoridad monetaria será exitosa en su afán por la estabilidad de precios, más fácilmente ajustarán a la baja sus expectativas inflacionarias.

Con base en lo anterior, queda claro que la independencia de la banca central, por si misma, no garantiza el triunfo en la lucha contra la inflación, ni que su ausencia necesariamente redunde en mayor inflación. Así, puede suceder que aún sin haberse realizado las modificaciones legales que le otorguen una plena autonomía a la autoridad monetaria, la sociedad en su conjunto puede estar convencida de los efectos nocivos que la inflación trae consigo, y por consiguiente, propiciar un entorno caracterizado por la estabilidad de precios.

La complejidad del proceso inflacionario y de su erradicación, somete a la autoridad monetaria a operar en un entorno por demás difícil. El banco central enfrenta el reto de abatir la

\footnotetext{
${ }^{7}$ Friedman (1963).

${ }^{8}$ Ver por ejemplo, Ball (1994).
} 
inflación, adquirir credibilidad y reputación de autoridad comprometida con dicha meta, en un entorno en el que factores ajenos a su control, juegan un papel importante en la determinación de la inflación. Más aún, como se señaló con anterioridad, las autoridades monetarias enfrentan la complicación adicional de que sus acciones de política afectan con rezago la evolución de los precios. Ante este escenario, la transparencia en la instrumentación de la política monetaria y en sus objetivos, resulta crucial para lograr una mayor credibilidad y ser así más exitosos en la disminución de la inflación.

La independencia de la banca central busca otorgarle a la autoridad monetaria la infraestructura legal necesaria para que la política monetaria conjunte el rigor y la disciplina con cierta flexibilidad que le permita responder a perturbaciones no anticipadas, sin que dicha discreción en la instrumentación de la política, redunde en incertidumbre y en el sesgo inflacionario que caracteriza a las políticas discrecionales. Como se mostrará más adelante, este arreglo institucional permite que el banco central enfrente satisfactoriamente el problema de la "incongruencia dinámica" que surge cuando la política monetaria cuenta con cierto grado de discrecionalidad. Dicho de otra manera, la independencia le otorga flexibilidad a la autoridad monetaria y elimina los problemas de verse obligada a adoptar reglas específicas que impidan la reacción oportuna de la política ante la aparición de eventos no anticipados.

Lo anterior implica que la introducción de un arreglo institucional, tal como la independencia de la banca central, mejora la credibilidad de la política monetaria, y por lo tanto favorece la disminución de la inflación. Sin embargo, la contribución de esta reforma legal será más valiosa mientras más convencida se encuentre la sociedad de que la estabilidad de precios es un fin digno de lograrse.

\section{Sustento Teórico a Favor de la Independencia de la Banca Central}

En esta sección se describen los principales avances teóricos que han dado sustento a la independencia de las autoridades monetarias. La primera parte de esta sección presenta algunos conceptos básicos sobre la credibilidad en las políticas económicas y la llamada "incongruencia intertemporal" de las políticas económicas. La introducción de estos conceptos resulta fundamental para el desarrollo de los modelos de reputación y preferencias de la autoridad monetaria que se presentan en el segundo apartado de esta sección, y para la descripción de los modelos de independencia de la banca central que aparecen posteriormente. 


\section{III.1. Reglas vs Discreción en la Política Monetaria y la "Incongruencia Intertemporal”}

El trabajo de Kydland y Prescott (1977) es tal vez el más citado en el tema de credibilidad, y se ha constituido en una pieza fundamental para el desarrollo de diversas ramas de la literatura económica. Dicho trabajo presenta una clara descripción del conflicto que enfrentan las autoridades para la instrumentación de políticas óptimas en distintos períodos de tiempo, la reacción del público ante esta disyuntiva, y la forma en que la interacción entre la autoridad y el público en las circunstancias descritas, puede llevar a un equilibrio no óptimo9 .

El trabajo de Kydland y Prescott, del cual se derivan una serie de modelos teóricos que abordan el tema de la independencia de la banca central, toma como punto de partida el concepto de "incongruencia intertemporal" ${ }^{10}$. Esta idea se refiere a la incongruencia entre las políticas óptimas que la autoridad anunciaría si sus anuncios fueran creídos por el público, y las políticas que la autoridad lleve a cabo una vez que el público ha actuado con base en esas expectativas. Las autoridades pueden tener cierta inclinación a tomar ventaja de la posible existencia de una curva de Phillips de corto plazo, que presente una relación inversa entre desempleo e inflación, para así disminuir el desempleo. Esto ocurre debido a que las sorpresas inflacionarias pueden generar un menor desempleo. La idea de que tanto el nivel de empleo como el nivel de producto se ven afectados ante la presencia de una sorpresa inflacionaria, es decir ante una expansión en la oferta de dinero no esperada, lleva a que la política monetaria tenga un sesgo inflacionario.

En el modelo de Kydland y Prescott la política óptima exante de la autoridad monetaria es realizar un anuncio mediante el cual se establezca su compromiso con una política antiinflacionaria. Sin embargo, esta política no satisface la optimalidad expost, ya que si el público responde aceptando contratos salariales con una expectativa de inflación a la baja, las autoridades tendrán el incentivo de generar una sorpresa inflacionaria, definida ésta como la diferencia entre la inflación observada y la anticipada al momento de acordarse los contratos laborales, y lograr con ello una mayor disminución en el desempleo y pasar así de un equilibrio de "segundo mejor"11 a uno de "primero mejor" ${ }^{12}$. No obstante, debido a que el público

\footnotetext{
${ }^{9}$ Algunos otros modelos dinámicos que también presentan problemas de credibilidad aplicados a distintas ramas de la economía son: Auernheimer (1974), Calvo (1978), Eichengreen (1990) y Fischer (1980).

${ }^{10}$ También llamada "incongruencia dinámica".

${ }^{11}$ Conocido como "second best" en la literatura económica.
} 
anticipa la posibilidad de que se presente una sorpresa inflacionaria, éste ajustará su comportamiento con base en dicha expectativa, estableciendo salarios más elevados en sus contratos de trabajo, y afectando así la determinación de los precios. El resultado final será un equilibrio de "tercero mejor"13.

En forma general, los problemas de credibilidad en la política económica pueden describirse de la siguiente manera: supongamos que una autoridad quiere diseñar en el período (t) una regla de política óptima para un cierto período en el futuro $(t+s)$. La autoridad determina dicha política a través de la maximización de alguna función objetivo, sujeto a las restricciones que enfrente, en función, por ejemplo, del comportamiento del público. Así, una consideración importante para que la autoridad determine en el período (t) su política óptima para el período $(\mathrm{t}+\mathrm{s})$, será como la expectativa de que se lleve a cabo dicha política en $(\mathrm{t}+\mathrm{s})$ afecte las decisiones del público en el intervalo de tiempo entre los períodos $(\mathrm{t})$ y $(\mathrm{t}+\mathrm{s})$. El comportamiento del público en el intervalo de tiempo entre el período $(\mathrm{t})$ y $(\mathrm{t}+\mathrm{s})$ dependerá de si éste considera o no creíble el anuncio de la autoridad realizado en ( $\mathrm{t}$ ) de llevar a cabo la política señalada en el período $(t+s)$. En el caso de que el público se encuentre convencido de que en ( $t+s)$ la autoridad llevará a cabo la política anunciada en $(t)$, una vez alcanzado el período $(\mathrm{t}+\mathrm{s})$, podría suceder que el plan que formuló la autoridad en $(\mathrm{t})$, con base a las restricciones en ese entonces presentes, ya no resulte óptimo, una vez que se conocen las nuevas restricciones, es decir el comportamiento del público en el lapso de tiempo entre (t) y $(t+s)$. Ante esta situación, la maximización de la función objetivo de la autoridad, sujeta a las nuevas restricciones determinará una política óptima distinta a la determinada en el período (t). Así, el plan original resulta "incongruente en el tiempo".

Esta "incongruencia intertemporal" de las políticas caracteriza un amplio rango de problemas económicos. No obstante, para que dicha incongruencia se presente se requiere que la autoridad en (t) enfrente una situación de "segundo mejor", pues si en (t) la autoridad ya alcanzó la optimalidad de "primero mejor", no habrá razón para desviarse del plan original. Sin embargo, si el público anticipa que la autoridad no cumplirá su anuncio en el período $(\mathrm{t}+\mathrm{s})$, su comportamiento determinará las nuevas restricciones que la autoridad enfrente al maximizar su función objetivo en ( $t+s)$. La política resultante será inferior en bienestar a la que se obtendría tanto en el caso de que la autoridad cumpliera con su anuncio, o bien en el caso de incumplimiento de la autoridad pero de sorpresa para el público, el cual anticipaba el cumplimiento del anuncio.

\footnotetext{
${ }^{12}$ Conocido como "first best" en la literatura económica.

${ }^{13}$ Conocido como "third best" en la literatura económica.
} 
Así, mientras el público anticipe que la autoridad (la cual cuenta con la discreción para revisar secuencialmente su política y se encuentre en una situación de "segundo mejor") tendrá incentivos para incumplir sus anuncios de política con el objeto de alcanzar un "primero mejor", éste ajustará su comportamiento con base en dicha expectativa, lo cual resultará en una solución de "tercero mejor".

El problema descrito, para el caso específico de la política monetaria se presenta de manera esquemática en la siguiente gráfica.

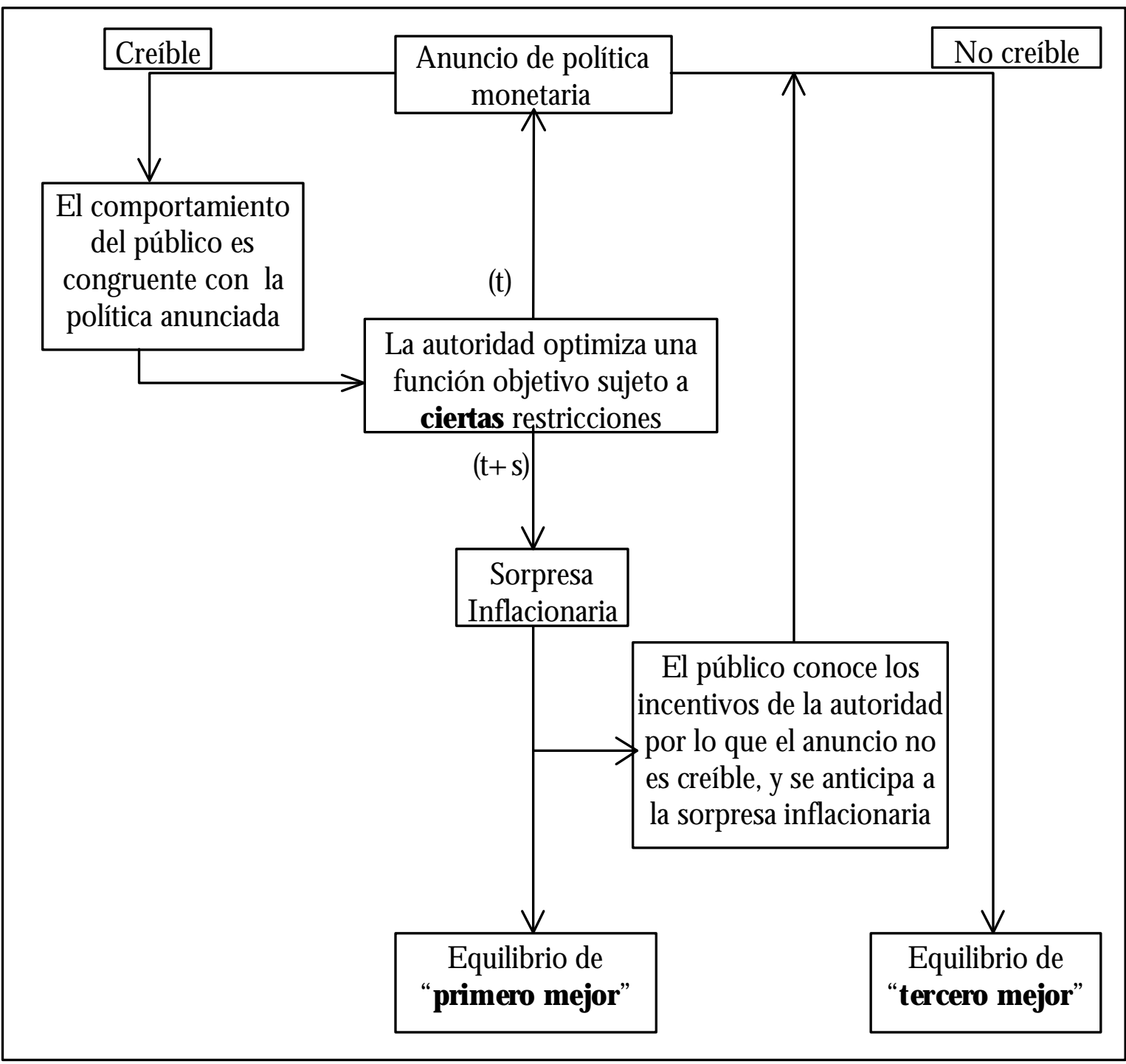

La secuencia de eventos inicia en el recuadro central en el que la autoridad monetaria optimiza cierta función objetivo, sujeto a ciertas restricciones. Con base en dicho proceso de 
optimización, la autoridad realiza en el período (t) un anuncio sobre su política monetaria. Dicho anuncio puede resultar creíble o no creíble. En caso de que el anuncio de política resulte creíble, el comportamiento del público será congruente con la política monetaria anunciada. Ante tal situación, en el período $(t+s)$, la autoridad monetaria volverá a optimizar su función objetivo sujeto a restricciones distintas a las que enfrentó en (t). Como resultado de dicha optimización, la autoridad monetaria tendrá el incentivo a incumplir su anuncio, generando una sorpresa inflacionaria y pasar así de un equilibrio de "segundo mejor" a uno de "primero mejor".

Sin embargo, ante la posibilidad de que la autoridad incumpla con su anuncio, el público no creerá en el anuncio de la autoridad, e intentará anticiparse a la sorpresa inflacionaria. Dicha acción resultará en un equilibrio de "tercero mejor", lo cual representa una situación de bienestar social inferior a las anteriores.

Supongamos ahora que la autoridad pudiera realizar un compromiso estricto en (t), y no solamente un anuncio, de que llevará a cabo una política específica en $(t+s)$. Dicho compromiso podría ser creíble si por ejemplo, el costo del incumplimiento fuera muy elevado. Ante esta situación, en la que se contaría con una "tecnología de compromiso", resultaría irrelevante el problema de la "incongruencia temporal". Sin embargo, en realidad las autoridades difícilmente tienen acceso a dichas tecnologías, por lo que operan en un ambiente de política discrecional en el que las políticas se encuentran sujetas a revisiones. La posibilidad de que las políticas se revisen y las autoridades incumplan con sus anuncios de política, propicia que los agentes económicos ajusten su comportamiento a dichos eventos, lo cual redunda en ineficiencias y en elevados costos para la sociedad.

La literatura posterior al artículo de Kydland y Prescott, que ya toma como base los problemas de "incongruencia intertemporal", presenta soluciones a este problema tratando de introducir las llamadas "tecnologías de compromiso".

En el modelo de Kydland y Prescott se asume que los bancos centrales diseñan su política monetaria minimizando una función de pérdida de bienestar social, la cual contempla la posibilidad de obtener menores niveles de desempleo a cambio de una inflación mayor a la anticipada por el público. El único instrumento bajo el control de la autoridad monetaria es la oferta monetaria, la cual determina el nivel de inflación. D e esta manera, la autoridad genera 0 crea sorpresas inflacionarias expandiendo la oferta monetaria por encima de lo esperado. La función de pérdida presenta parámetros que reflejan la importancia relativa que asigna la 
autoridad monetaria a los costos de un mayor nivel de inflación y a los beneficios de un menor desempleo.

En el modelo descrito, si bien la autoridad presenta cierta aversión a la inflación, los beneficios marginales de un menor desempleo, como consecuencia de una sorpresa inflacionaria, sobrepasan los costos de la mayor inflación, por lo que la autoridad estará dispuesta a generar la sorpresa inflacionaria. El equilibrio en este modelo se alcanza en una situación en la cual la tasa de inflación es lo suficientemente alta que el costo marginal de generar una sorpresa inflacionaria es igual al beneficio marginal del menor desempleo que produciría. Así, en equilibrio, la inflación resulta excesiva. Dicho resultado ocurre por el hecho de que la autoridad no puede hacer un anuncio creíble de que en el futuro actuará de acuerdo a los planes originales. Con base en el resultado del modelo, resulta claro que cualquier fórmula 0 arreglo institucional que permita comprometer a la autoridad a no crear sorpresas inflacionarias, reducirá el nivel de equilibrio de la inflación.

En una primera instancia, la literatura sugirió resolver los problemas de "incongruencia intertemporal" que surgen por la discrecionalidad de la política monetaria y que dan lugar a sesgos inflacionarios, mediante la adopción de una regla monetaria. Es decir, la literatura proponía abandonar las políticas monetarias discrecionales y substituir éstas por reglas precisas para la instrumentación de dichas políticas. Por ejemplo, la adopción de una regla monetaria tal como la sugerida por Friedman (1959), en la que la oferta monetaria crece a una tasa constante, evitaría que la autoridad expanda la oferta monetaria con el objeto de generar sorpresas inflacionarias. Así la regla forzaría a que la cantidad de dinero aumente a una tasa que no genere inflación y que por consiguiente resulte en la estabilidad de precios. No obstante, el estricto seguimiento de reglas como la señalada impide que la autoridad actúe oportunamente instrumentando las medidas necesarias para hacer frente a situaciones no previstas. Más aún, no existe garantía de que la autoridad no abandone la regla en el futuro, por lo que no es del todo claro que la adopción de una regla monetaria lograría reducir el sesgo inflacionario que aparece en los modelos originales.

El hecho de que el equilibrio resultante de una política monetaria discrecional genere un sesgo inflacionario, hace que dicho equilibrio no sea óptimo para la sociedad en su conjunto. Ante este resultado surge el siguiente cuestionamiento: ¿Cómo puede modificarse el marco institucional en el cual opera la política monetaria para eliminar el sesgo inflacionario que ésta genera cuando se instrumenta de manera discrecional?. Con el propósito de responder a esta interrogante y solucionar el problema de "incongruencia intertemporal" en la política monetaria, se han desarrollado distintos modelos que proponen mecanismos alternativos a 
través de los cuales los bancos centrales puedan instrumentar una política monetaria que sea congruente en el tiempo y que conlleve a un bajo nivel de inflación. Dicho de otra manera, la credibilidad en los anuncios de política de la autoridad monetaria dependerá de sí dichas políticas son "congruentes en el tiempo".

Estos modelos pueden dividirse en dos grupos: i) aquéllos que hacen referencia a la relación de largo plazo que existe entre los bancos centrales y el público, y como el mantener cierta reputación ante el público genera los incentivos para que las autoridades mantengan una política monetaria orientada hacia la consecución de equilibrios con bajos niveles de inflación (modelos de reputación y preferencias de la autoridad monetaria); y ii) aquéllos que crean arreglos institucionales que logran modificar los incentivos de las autoridades monetarias de tal forma que éstas encuentran como su mejor estrategia procurar la estabilidad de precios (modelos de independencia de la autoridad monetaria). A continuación se presentan las principales características de los modelos que se han desarrollado con el objeto de solucionar el problema de la "incongruencia intertemporal" de la política monetaria.

\section{III.2. Modelos de Reputación y Preferencias de la Autoridad Monetaria}

Basados en la idea de que la instrumentación de cualquier política representa una interacción repetida entre la autoridad monetaria y el público, la política instrumentada por las autoridades en un momento determinado genera un vínculo con las políticas que pudieran llevarse a cabo en el futuro. Así, desviaciones de la autoridad de la política anunciada no solo pueden implicar ciertos beneficios en el presente, sino también costos en el futuro. La existencia de costos futuros disminuye los incentivos de la autoridad a incumplir sus anuncios de política, razón por la cual las políticas anunciadas se vuelven más creíbles. Con base en esta idea surge la conveniencia de que la autoridad goce de reputación antiinflacionaria, lo cual redundaría, aún en el caso de un entorno caracterizado por la instrumentación de políticas discrecionales por parte de la autoridad, en la instrumentación de políticas que resulten en un mayor beneficio para la sociedad.

D e esta manera, los modelos de reputación permiten que aún con problemas de credibilidad, las políticas antiinflacionarias prevalecen en el equilibrio. Esto ocurre por el hecho de que la autoridad esta consiente de que el incumplimiento de sus anuncios de política, le representará la aparición de costos que a la postre le dificultarán la instrumentación de políticas que juzgue óptimas. 
Con base en lo recién señalado, Barro y Gordon (1983) sugieren solucionar el problema de credibilidad adaptando un modelo de construcción de reputación del tipo utilizado en organización industrial $^{14}$. De manera específica, estos autores proponen que si el público penaliza a la autoridad monetaria por desviarse de sus anuncios de política, disminuyendo su credibilidad en los anuncios futuros, es posible mantener a la inflación y a las expectativas de inflación en un equilibrio más eficiente que el que resulta del modelo de Kydland y Prescott ${ }^{15}$.

Los modelos de reputación se basan en la idea de que las autoridades monetarias y el público interactúan varios períodos. Por consiguiente, las expectativas de los agentes se forman de la siguiente manera: cuando en un determinado período el banco central instrumenta la política monetaria que había anunciado previamente, en el siguiente período de interacción entre el público y la autoridad monetaria, el público creerá el anuncio de las autoridades y ajustará su comportamiento en función de la política anunciada. Por el contrario, si la autoridad incumple su anuncio y crea una sorpresa inflacionaria, en el período siguiente el público no considerará el anuncio de la autoridad y se obtendrá un equilibrio de alta inflación.

Continuando con la lógica de estos modelos, asumamos que el banco central ha sido una autoridad que ha cumplido con sus anuncios de política monetaria, los cuales se han encaminado a abatir el proceso inflacionario. Así, la decisión entre persistir con una política antiinflacionaria o generar una sorpresa inflacionaria dependerá de la comparación entre los beneficios que obtenga por crear la sorpresa inflacionaria y los costos en que incurra en el futuro por la pérdida de credibilidad. Dicha pérdida de credibilidad se reflejará en que el público anticipará un mayor nivel de inflación, el cual resulta equivalente al que se obtiene en un modelo de discreción en la instrumentación de la política monetaria que carezca de algún tipo de "tecnología de compromiso".

Este tipo de modelos muestra como un mecanismo de reputación puede aminorar el sesgo inflacionario de una política monetaria que cuenta con cierta flexibilidad y discreción para enfrentar perturbaciones no anticipadas. El que la efectividad de las acciones por parte de los bancos centrales dependa de la credibilidad que el público tenga en la autoridad monetaria, propicia que el banco central, con el afán de reforzar su reputación, continúe instrumentando políticas orientadas a equilibrios de baja inflación. En la práctica, la reputación de los bancos centrales se encuentra estrechamente ligada con el grado de independencia que éstos gocen de las demás instancias gubernamentales. Por ello, este tipo de modelos arroja argumentos

\footnotetext{
${ }^{14}$ Milgrom y Roberts (1982).

${ }^{15}$ Barro (1986), Canzoneri (1985) y Chari y Kehoe (1990) presentan otros modelos de reputación.
} 
formales a favor de una mayor autonomía de los bancos centrales y sobre todo, de una mayor independencia en el diseño de la política monetaria.

O tra solución al problema de credibilidad la ofrece Backus y Driffil (1985), adaptando el modelo de organización industrial de Kreps y Wilson (1982). La idea principal del modelo de Backus y Driffil es que a lo largo del tiempo las autoridades monetarias modifican sus preferencias con respecto a su aversión a la inflación, razón por la cual el público no necesariamente conocerá las preferencias de la autoridad en un momento determinado. Ante el escenario descrito, el público tratará de inferir el grado de aversión a la inflación de la autoridad, a través del tipo de políticas que el banco central lleve a cabo.

Por su parte, la autoridad monetaria tendrá el incentivo de ser percibida por el público como muy adversa a la inflación. En caso de que la autoridad en verdad sea sumamente adversa a la inflación, ésta se encontrará dispuesta a tolerar altas tasas de desempleo, con el objeto de mantener baja la inflación. Sin embargo, aún cuando la autoridad monetaria no sea adversa a la inflación, ésta buscará aparentar dicha aversión, y por consiguiente estará dispuesta a tolerar cierto nivel de desempleo. Esto último debido a que si la autoridad logra ser percibida como antiinflacionaria, entonces podrá ser capaz de convencer al público de dicha aversión y disminuir así sus expectativas de inflación, para después poder generar una sorpresa inflacionaria y obtener ganancias importantes en la reducción del desempleo. De esta manera, al tolerar altos niveles de desempleo, la autoridad monetaria, sin importar su grado de aversión a la inflación, irá construyendo su reputación como intolerante hacia el proceso inflacionario.

En el equilibrio de este modelo, la probabilidad que el público le asigne a la autoridad de ser adversa a la inflación será mayor conforme más alto resulte ser el nivel de desempleo. El resultado de este análisis es que aumentar la credibilidad de la autoridad es costoso en términos del empleo. Dicho de otra manera, la credibilidad en la autoridad monetaria se obtiene a través de las acciones que ésta lleve a cabo. Otra implicación del modelo es que al resultar costoso el abatimiento de la inflación, podría alcanzarse un equilibrio con alta inflación y un bajo nivel de desempleo, sin que la autoridad monetaria considere mejorar su credibilidad. Por su parte, si la autoridad monetaria llega a ser reconocida por su gran aversión a la inflación, una extensión natural al modelo de Backus y Drifill sería la incorporación de alguna "tecnología de compromiso", tal como la independencia de la banca central.

\section{III.3. Modelos de Independencia de la Autoridad Monetaria}


Q uizás el artículo del cual se deriva de manera más explícita la conveniencia de otorgarle independencia a la autoridad monetaria, para resolver el problema de credibilidad es el de Rogoff (1985). Este autor sugiere que la sociedad puede beneficiarse si el banco central es reconocido por asignarle mayor importancia a la reducción de la inflación que a la del desempleo en su función objetivo. Así, la independencia de la autoridad monetaria mitiga el problema de credibilidad, al otorgarle la instrumentación de la política monetaria a individuos (banqueros centrales conservadores) que se caracterizan por presentar una gran aversión a la inflación ${ }^{16}$. Esto ocurre debido a que el público, al conocer las preferencias del banquero central conservador e inferir que la política monetaria tendrá como objetivo prioritario abatir el crecimiento de los precios, disminuirá sus expectativas de inflación y por consiguiente sus demandas de ajustes salariales al alza.

Como el banco central en el modelo de Rogoff es adverso a la inflación, el equilibrio se alcanza en una situación en la que la inflación es menor que en el caso en que la autoridad monetaria mantiene la discreción en la instrumentación de su política, pero carece de la "tecnología de compromiso" (en este caso la presencia de un banquero central conservador). No obstante, en el modelo de Rogoff la autoridad conserva la posibilidad de poder reaccionar de manera discrecional ante perturbaciones inesperadas ${ }^{17}$.

De esta manera, aún en un entorno caracterizado por la instrumentación de una política monetaria discrecional que le permita a la autoridad hacer frente a perturbaciones no anticipadas, la política antiinflacionaria del banco central será creíble. Dicho de otra manera, la independencia del banco central evita que el exceso de discreción con el que cuente la autoridad monetaria para enfrentar un entorno cambiante e incierto, redunde en un equilibrio caracterizado por sesgos inflacionarios, que resulten por la falta de credibilidad en la política antiinflacionaria de la autoridad.

De los modelos teóricos analizados queda claro que la introducción de "tecnologías de compromiso", tales como cambios institucionales que impliquen la independencia del banco central, aumenta la credibilidad. Por su parte, la mayor credibilidad ayuda a disminuir la inflación a un menor costo. No obstante, para que los modelos alcancen dicha conclusión, el

\footnotetext{
${ }^{16}$ Una alternativa a la imposición de un banquero central conservador con el objeto de eliminar el sesgo inflacionario de una política monetaria discrecional, la proponen Persson y Tabellini (1993) y Walsh (1995). Estos autores sugieren que el propio contrato de remuneración del banquero central lo desincentive a generar inflación.

${ }^{17}$ El modelo de Rogoff ha sido extendido en diversas direcciones entre las que destacan la interacción entre la política fiscal y monetaria. Ver por ejemplo, Lohmann (1992) y D ebelle y Fischer (1995).
} 
marco teórico utilizado debe ser tal que el proceso de formación de expectativas del público considere, al menos parcialmente, las políticas futuras de la autoridad, pues de otra manera no surgirían los problemas de "incongruencia dinámica". Esto resulta importante, ya que de no presentarse esta condición, la pura expectativa de que disminuya la inflación propiciará denominar "creíble" a cualquier política que se este instrumentando, aunque dicha política no este necesariamente encaminada a disminuir la inflación. Por ejemplo, en países con baja inflación, es factible seguir observando menores niveles de inflación y que las expectativas se ajusten a dicha expectativa, sin la presencia forzosa de políticas antiinflacionarias. 


\section{Conclusiones}

La independencia de la banca central es un elemento que propicia un adecuado manejo monetario, y por consiguiente favorece la reducción de la inflación. La introducción de este arreglo institucional permite resolver los problemas de "incongruencia intertemporal" y de "sesgo inflacionario" que se presentan cuando la política monetaria cuenta con cierta flexibilidad para enfrentar situaciones no previstas. No obstante, el proceso inflacionario es por demás complicado, por lo que la instrumentación de una política monetaria prudente resulta crucial, más no suficiente, para alcanzar la estabilidad de precios.

La instrumentación de la política monetaria puede describirse como un proceso continuo de interacción entra las autoridades y el público. La teoría económica ha logrado avances importantes en el entendimiento de los incentivos, respuesta y comportamiento, tanto de la autoridad monetaria como del público en general. Así, la independencia de la banca central no ha sido tan sólo un acontecimiento empírico que ha tomado relevancia durante los últimos años, sino que se deriva directamente de la propia teoría económica.

El banco central enfrenta el reto de abatir la inflación, adquirir credibilidad y reputación de autoridad comprometida con la lucha antiinflacionaria, en un entorno en el que, en el corto plazo, factores distintos a los monetarios, y por lo tanto ajenos a su control, pueden incidir en forma importante sobre la evolución de los precios. Más aún, la política monetaria enfrenta la dificultad adicional de que sus acciones actúan con cierto rezago sobre el comportamiento de los precios. Ante el escenario descrito, tanto la teoría económica como la evidencia empírica, sugieren los siguientes elementos para mejorar la credibilidad de la banca central, y por consiguiente, facilitar el proceso de abatimiento de la inflación:

- El banco central debe tener un mandato claro y definido orientado a procurar la estabilidad de precios;

- La autoridad monetaria debe anunciar públicamente sus objetivos, y los mecanismos con los que pretende alcanzar dichas metas;

- La instrumentación de la política monetaria debe realizarse, en lo posible, a través de mecanismos de mercado;

- El banco central debe responsabilizarse de sus actos y justificar su proceder ante las instancias legislativas y el público en general;

- Con el objeto de poder alcanzar sus metas, la autoridad monetaria debe gozar de "independencia de instrumentos"; 
- Las acciones de política oportunas del banco central resultan fundamentales para afectar las expectativas inflacionarias del público, y por lo tanto facilitan el abatimiento de la inflación;

- La credibilidad en la política monetaria se logra a través de la instrumentación sostenida de políticas encaminadas a la reducción de la inflación; y

- Entre mayor sea el consenso de la sociedad y del resto de las políticas económicas de la relevancia de alcanzar la estabilidad de precios, más rápida y menos costosa será su consecución.

No cabe duda que los países más exitosos en la lucha contra la inflación han sido aquéllos que han seguido en mayor medida las recomendaciones señaladas. La independencia de la banca central representa un paso en la dirección adecuada para propiciar la reducción sostenible del ritmo de crecimiento de los precios. Sin embargo, dicho arreglo institucional debe complementarse con una serie de elementos que redunden en mayor credibilidad en la autoridad monetaria. El análisis de dichos elementos, ha sido el propósito de este trabajo. 


\section{Referencias}

Alesina, A. and L. Summers (1993), "Central Bank Independence and Macroeconomic Performance", Jaumal of MoneyCreeit andBanking Vd. 25, n 2.

Auernheimer, L. (1974), "The Honest Government Guide to the Revenue from the Creation of Money", Jaumal of Pditical Econamy, 82

Ball, L. (1994), "What D etermines the Sacrifice Ratio?", en Mankiw, N.G . (ed), MondaryPdig, NBER, Studies in Business Cycles, Vol. 29, Chicago.

Backus, D . and J. D riffil (1985), "Inflation and Reputation”, Ameican EconamicReiew 75.

Barro, R. J. (1986), "Reputation in a Model of Monetary Policy with Incomplete Information", Jaumal of Montary Economics 17.

--, and D. B. Gordon (1983), "Rules, Discretion and Reputation in a Model of Monetary Policy", Jaumal of Mondary Economics, 12

Blinder, A. S. (1998), "Survey Evidence on Central Bank Credibility", NBER- Universities Research Conference: Formulation of Monetary Policy, Cambridge, MA, D ecember.

Bruno, M. and W.R. Easterly (1995), "Inflation, Crisis, and Long-run Growth", NBER, WorkingPaper Seies, no 5209.

Calvo, G. (1978), "On the Time Consistency of Optimal Policy in a Monetary Economy", Economerica, 46.

Canzoneri, M. (1985), "Monetary Policy Games and the Role for Private Information", American EcommicReiew 75.

Capie, F., G oodhart, C., Fischer, S. and N. Schnadt (1994), "TheFutureof Central Banking", The Tercenary Symposium of the Bank of England, Cambridge University Press, G reat Britain.

Cecchetti, S. G. (1998), "Policy Rules and Targets: Framing the Central Banker's Problem", Fedkal ReserveBank of NewYok, EconomicPdioy Reiew June.

Chari, V.V. and P. J. Kehoe (1990), "Sustainable Plans", Jamal of Pditical Economy, Vd. 98, n 4.

Cukierman, A . (1992), Cetral Bank Strategy, Credbility, and Independence Thery and Evidence MIT Press, London. 
D ebelle, G. and S. Fischer (1995), "How Independent should a Central Bank Be?", in J.C. Fuhrer (ed), G oals, Guiddines and ConstrainingFaaingMondary Pdigmakes Federal Reserve Bank of Boston, Conference Series, $n^{\circ}$ 38, Boston.

Drazen, A. and P. R. Masson (1994), "Credibility of Policies versus Credibility of Policy Makers", IMF WorkingPaper mo 49, May.

Eichengreen, B. (1990), "The Capital Levy in Theory and Practice", en D ornbusch, R., and M. D raghi (eds), PublicDedt Managenent: TheryandHistary, CEPR, Cambridge.

Eijffinger, S. and E. Schaling (1995), "O ptimal Commitment in an O pen Economy: Credibility vs Flexibility”, Bank of Engand, WorkingPaper no 41, D ecember.

Faust, J. and L.E.O Svensson (1998), "Transparency and Credibility: Monetary Policy with Unobservable G oals", NBER WorkingPapern ${ }^{\circ}$ 6452, March.

Fischer, S. (1980), "Dynamic Inconsistency, Cooperation, and the Benevolent Dissembling G overnment", Jaumal of EconomicDynamics and Contrd, 2

--, (1990), "Rules versus Discretion in Monetary Policy" en Friedman, B. M. and F. H. Hahn (eds), Handbodk of MontaryEconomics Vd. II.

--, (1993), The Role of Macroeconomic Factors in Growth", Jaumal of MondaryEconamics, 32.

Forder, J. (1998), "Central Bank Independence, Conceptual Clarifications and Interim Assessment", OxfordEconmicPapes, 50.

Friedman, M. (1959), A Progamfor Montary Stability, Fordham University Press, New Y ork.

--, (1963), "Inflation: Causes and Consequences", The Camil for Econamic Eduration, Asia PublishingHase, Bombay.

Kreps, D. and R. Wilson, (1982), " Reputation and Imperfect Information”, Jamal of Economic Thery, 27.

Kydland, F.E. and E. C. Prescott (1977), "Rules Rather than D iscretion: The Inconsistency of O ptimal Plans", Jaumal of Pditical Economy, 85.

Lohmann, S. (1992), "Optimal Commitment in Monetary Policy: Credibility versus Flexibility”, TheAmerican EconamicReriew 82 
Martínez, L. (1998), "El Efecto de la Inflación en la Distribución del Ingreso", Doumento Nº. 9806, SeieDoumentos deInvetigaión Banco de México.

Milgrom, P., and J. Roberts (1982), "Limit Pricing and Entry under Incomplete Information", Econamenica, 50.

Perrson, T., and G. Tabellini, (1993), "D esigning Institutions for Monetary Stability", Camege Rochester Series on PublicPdioy, 39.

--, (1994), MondaryandFiscal Pdig, Vd. 1: Creeliblity, MIT Press, Cambridge, London.

Posen, A. (1998), "Central Bank Independence and Disinflationary Credibility: A Missing Link?", OxfordEconomicPapes, 50.

Rogoff, K. (1985), " The Optimal Degree of Commitment to an Intermediate Monetary Target", QuartelyJaumal of Econmiss, 100.

Viñals, J. (1998), "The Retreat of Inflation and the Making of Monetary Policy: Where Do We Stand?", DoumentodeTrabajon ${ }^{\circ}$ 9813, Banco de España.

--, (1998), “Monetary Policy and Inflation”, DisassionPapern n 1821, CEPR, February.

Walsh, C.E. (1995), "O ptimal Contracts for Central Bankers", Amtican EconomicReriew 85. 